BULLETIN (New Series) OF THE

AMERICAN MATHEMATICAL SOCIETY

Volume 40, Number 1, Pages 69-87

S 0273-0979(02)00967-9

Article electronically published on October 15, 2002

\title{
SOME MATHEMATICAL CHALLENGES IN MATERIALS SCIENCE
}

\author{
JEAN E. TAYLOR
}

\begin{abstract}
Four challenges to mathematics research posed by the field of materials science are given, plus an additional challenge purely from the field of geometric measure theory. The problems all concern the effects of surface and grain boundary free energy: motion by weighted mean curvature and/or surface diffusion and/or other kinetics, proofs of minimality of soap bubble clusters and their anisotropic analogs, shapes of crystals in a gravitational or other field, incorporating data from simulations into mathematics, and understanding flat chains modulo $\nu$. The figures are selected copies of transparencies presented at the lecture on which this paper is based.
\end{abstract}

Materials science is both much older and much younger than mathematics. Ancient humans discovered various elemental metals, like gold and copper. At some point, they discovered that if you mix a little tin into copper, you get something quite different from either, namely bronze. There is a period of human history labeled the Bronze Age; I don't think there is any labeled the Arithmetic Age.

On the other hand, the term "materials science" only came into general use within the last 30 years or so, as the subjects of metallurgy, ceramics, and polymers were collected into one discipline. The Materials Research Society was founded in 1973, and the International Congress of Materials Research Societies in 1991. And along with the old problems of making better structural materials such as steel came new issues unimaginable a century ago. Some of these include the ability to see and manipulate individual atoms with an atomic force microscope, the study of carbon nanotubes, the fabrication of electronic chips, and the making of thin films for CD's and other storage media.

What are mathematicians doing in such a field? First of all, as Rodney Nichols said at the New York Academy of Sciences on June 5, 2000, "Much more than a few decades ago, there are mathematicians everywhere - and that is for the good." Secondly, there has been an effort by the National Science Foundation to encourage this interdisciplinary research. A search of the NSF database under the Division of Mathematical Sciences, performed in the year 2000, revealed 85 grants since January 1, 1997 with the words "materials science" in their abstracts. There have been several multi-million-dollar awards, co-sponsored by DARPA, for such cooperative efforts targeted at specific problems. Part of the result is that the collection of mathematicians working on problems from materials science has grown from isolated individuals prior to the mid-1980's, to a small community, and now

Received by the editors November 6, 2000, and, in revised form, February 21, 2002.

2000 Mathematics Subject Classification. Primary 74N20; Secondary 49Q20, 49Q15, 35R99, 53A17, 65K10. 
to the size that the Third SIAM Conference on Mathematical Aspects of Materials Science, held in May 2000, had about 250 speakers (approximately equally divided between mathematicians and materials scientists).

I give here a view of some of the challenges that lie in the interaction between mathematics and materials science, limited by the length of the talk on which this article is based and by my own interests.

1. The first challenge is fundamental to materials science.

Challenge 1. Make mathematical models of grain orientations and boundaries in polycrystals and how they evolve. Then use the models to determine how to control the boundaries in processing.

To explain this challenge, one must first understand the internal structure of metals, ceramics, and semiconductors. Materials are composed of many small crystals, called grains, each composed of atoms in a lattice or other regular arrangement. An interface between two grains is analogous to a film separating two bubbles of air in a soap froth, because it has an excess free energy (abbreviated hereafter as just "surface energy" or "energy"), similar to the surface area of the film. As in soap froths, there are triple junctions where three grains come together and point junctions shared by four grains, but there can also be more complicated junctions if the surface energies have the right relationships [HC].

The energy of the grain boundaries is due to atoms having "broken bonds" when compared to their arrangement in the interior of the crystal. Even if there is atomic rearrangement at and near the surface to decrease this excess energy, as can happen quite dramatically, an excess still exists. Furthermore, the structure of a surface (which may be an interface with another grain) is usually anisotropic, i.e., different

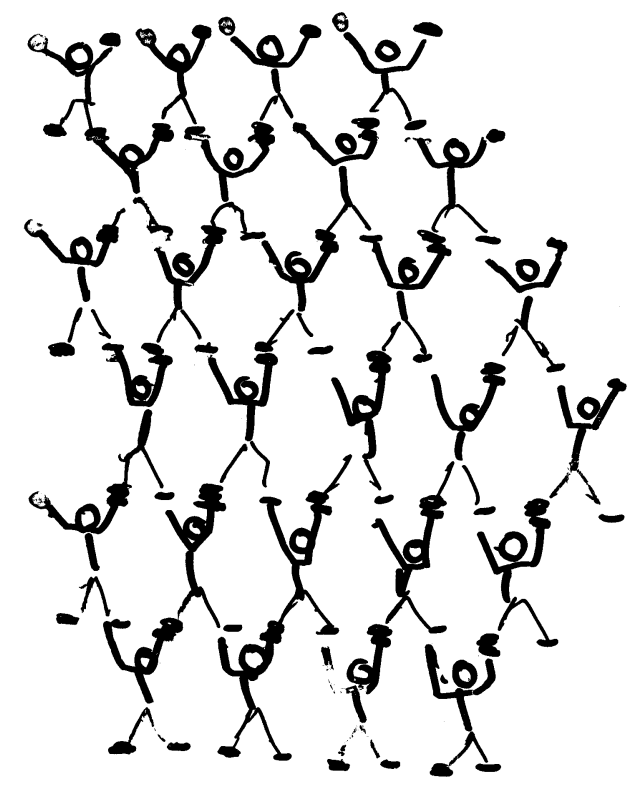

FiguRe 1. An illustration of anisotropic surface free energy due to broken bonds. 


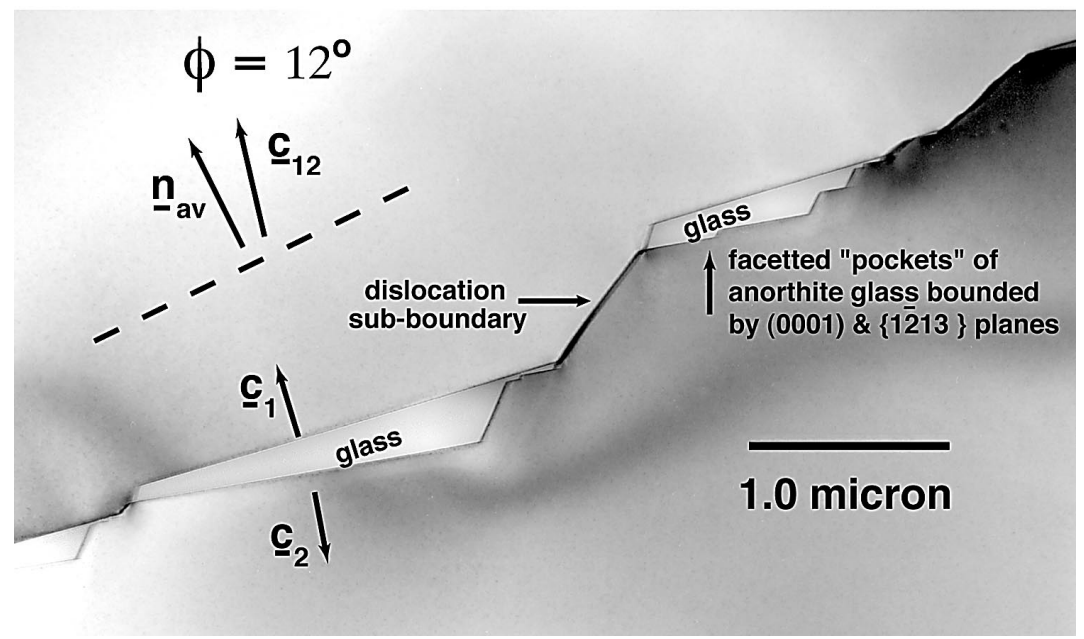

Figure 2. An illustration of a liquid layer occurring along an interface at some normal directions but not others. Courtesy of Carol Handerwerker, NIST, Gaithersburg, MD 20899.

in different normal directions. This is caricatured in the cartoon of Figure 1; there it is evident that some normal directions are "all hands," some are "all right hands," some are "left hands and left feet," etc. Thus the surface free energy per unit area, usually called $\gamma$ by materials scientists, is a function of the interface normal direction and the orientations of the crystals which meet along that interface. If the energy depends appropriately strongly on the normal direction, then local minimization of this energy can lead to the interfaces having facets and sharp angles rather than being smooth.

Interfaces in materials can also be vastly more complicated than what this suggests. Some materials have interfaces in which thin layers of a fluid phase occur between grains at certain normal directions to the interface but not others, and the crystal boundaries to each side of the fluid layer can have corrugations. These corrugations, and the alternation of wetted and non-wetted interfaces, can occur at arbitrarily small scales. This phenomenon occurs well below the bulk melting temperature, and the fluid layers can properly be thought of as infinitesimal; see Figure 2 and its reference. When there are composition differences, additional effects can occur; see Figure 3 for a high-resolution micrograph of a lead-cadmium inclusion embedded in an aluminum grain, where there are slabs of cadmium attached to facets of two particular (and opposite) normal directions of the lead particle. In this paper, all such details of interface structure will be subsumed into a single given surface energy function for each interface, depending on the normal directions of that interface and the orientations of the crystals meeting there.

Although it will not be treated here, there might also be an energy per unit length associated to triple junctions, and even a point energy associated to point junctions. If such an energy is positive, it is possible to include it in a mathematical model (see, for example, Mor94]), but if it is negative, it has the mathematically unreasonable property that increasingly wiggly junction curves can have negative infinity as the limiting total energy. See [T99m] for a discussion of this subject. 


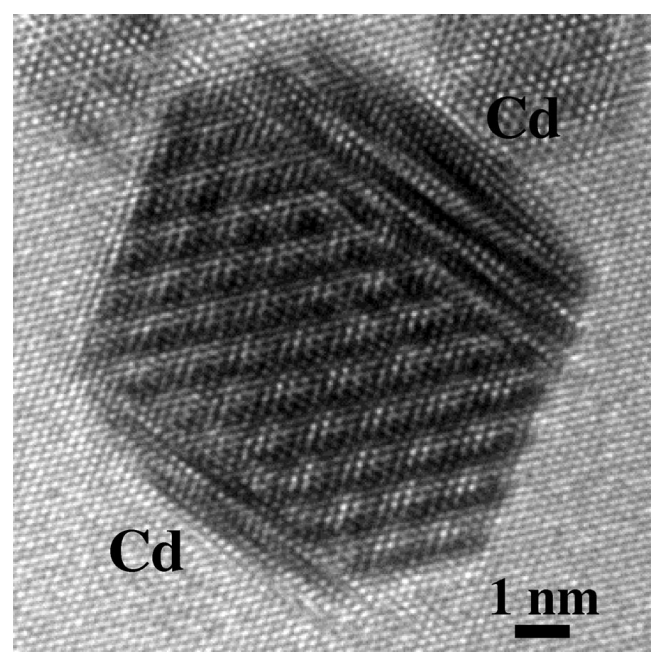

Figure 3. A lead crystal embedded in an aluminum crystal, with cadmium caps at each end. Reprinted with permission from E. Johnson and U. Dahmen, "In-situ Transmission Electron Microscopy Observations of Alloying in Nanoscale Pb Inclusions by Ion Implantation with Cd", Microscopy and Microanalysis $\mathbf{3}$ (1997), 409-416.

Another ingredient is a possible bulk free energy change per unit volume, denoted $\Omega$, which might either be assumed to be constant or might vary with position, perhaps though another variable such as temperature. An example of bulk free energy is the latent heat that is released when water freezes. A very important ingredient that is widely considered but that will not be considered here is elastic energy; all crystals will be assumed to be incompressible and undistorted.

In this review, we consider all crystals to have fixed orientations, and so for any given interface between a crystal with orientation labeled $\alpha$ and another (or a fluid) labeled $\beta, \gamma_{\alpha \beta}$ is a function of the oriented unit normal vectors. That is,

$$
\gamma_{\alpha \beta}:\left\{\mathbf{n} \in R^{3}:|\mathbf{n}|=1\right\} \rightarrow R^{+} .
$$

Except where we focus on polycrystals, we abbreviate $\gamma_{\alpha \beta}$ by $\gamma$. Furthermore, it is often useful to extend $\gamma$ by positive homogeneity - that is, to define $\gamma(r \mathbf{n})=r \gamma(\mathbf{n})$ for all $r \geq 0$, and thus have

$$
\gamma: R^{3} \rightarrow R^{+} \cup\{0\}, \quad \text { with } \quad \gamma(\mathbf{p})=|\mathbf{p}| \gamma(\mathbf{p} /|\mathbf{p}|), \quad \gamma(\mathbf{0})=0 .
$$

Surface free energy enters into equilibrium and growth laws via the weighted mean curvature $\kappa_{\gamma}$. If $\gamma$ is independent of normal direction, then $\kappa_{\gamma}$ is just $\gamma$ times the mean curvature $\kappa$. (Although mathematicians usually use $H$ for mean curvature, materials scientists reserve $H$ for enthalpy - heat content - and prefer to use $\kappa$ for both the curvature of curves and the mean curvature of surfaces.) In the anisotropic case, if $\gamma$ is smooth (say, $C^{3}$ ) and satisfies the convexity condition $\gamma\left(a_{1} \mathbf{n}_{1}+a_{2} \mathbf{n}_{2}\right)<a_{1} \gamma\left(\mathbf{n}_{1}\right)+a_{2} \gamma\left(\mathbf{n}_{2}\right)$, and if $S$ is a similarly smooth surface with oriented normal vector field $\mathbf{n}_{S}$, then $\kappa_{\gamma}$ is a weighted sum of the principal curvatures of $S$, where the weights are the second derivatives of (the homogeneously extended) 
$\gamma$ in the corresponding principal directions. As another way of expressing this,

$$
\kappa_{\gamma}(x)=\nabla_{S} \xi(x),
$$

where $\nabla_{S}$ denotes the surface divergence and $\xi$ is the Cahn-Hoffman [CH] vector

$$
\xi(x)=\nabla \gamma\left(\mathbf{n}_{S}(x)\right)
$$

(once again using the homogeneous extension of $\gamma$ to define $\nabla \gamma$ ).

A more useful way to regard $\kappa_{\gamma}$ is as the $L^{2}$ gradient of the surface free energy functional. That is, for a vector field $\mathbf{g}$ defined on an oriented surface $S$ and a real number $\lambda$, let $(\lambda \mathbf{g})_{\#} S$ be the surface

$$
(\lambda \mathbf{g})_{\#} S=\{x+\lambda \mathbf{g}(x): x \in S\},
$$

with the induced orientation. Define

$$
E\left((\lambda \mathbf{g})_{\#} S\right)=\int_{y \in(\lambda \mathbf{g})_{\#} S} \gamma\left(\mathbf{n}_{(\lambda \mathbf{g})_{\#} S}(y)\right) d \mathcal{H}^{2} y
$$

(with $\mathcal{H}^{2}$ denoting the usual Hausdorff area measure), and define

$$
\delta_{E} S(g)=\left.\frac{d E\left((\lambda \mathbf{g})_{\#} S\right)}{d \lambda}\right|_{\lambda=0} .
$$

If $\gamma$ is smooth and convex and if $S$ is a similarly smooth surface, then $\delta_{E} S(g)$ is a bounded linear functional on vector fields $\mathbf{g}$ which can be represented by integration. Its Radon-Nikodým derivative with respect to Hausdorff measure turns out to be $\kappa_{\gamma}$ :

$$
\delta_{E} S(g)=\int_{S} \mathbf{g} \cdot\left(\kappa_{\gamma} \mathbf{n}_{S}\right) d \mathcal{H}^{2} .
$$

Since the rate at which volume is swept out by deforming the surface by $\mathbf{g}$ is $\int_{S} \mathbf{g} \cdot \mathbf{n}_{S} d \mathcal{H}^{2}$, the weighted mean curvature can be thought of as $\frac{\delta E}{\delta V}$, the rate of change of energy with volume. This idea can be used to extend the definition of $\kappa_{\gamma}$ to cases where $\gamma$ is not differentiable and $S$ is not smooth. See [T78].

Other ingredients affecting crystal growth shapes are the mechanisms by which shapes can change. Two that will be discussed here include attachment and detachment kinetics, usually modeled via a mobility factor $M$ which can also depend on the normal direction, and diffusion of atoms over the surface, often modeled by a multiplicative constant or tensor $D$. Again, deformations of the grains themselves will not be considered here.

Letting $v(x)$ denote the velocity in direction $\mathbf{n}_{S}(x)$ at the point $x$ in $S$, the standard basic laws are then

(1) equilibrium with fixed boundary

$$
\kappa_{\gamma}=0
$$

(2) equilibrium with volume constraint

$$
\kappa_{\gamma}=C,
$$

(3) motion by mobility times a possibly zero-weighted mean curvature plus a possibly nonzero bulk free energy difference,

$$
v=M\left(\mathbf{n}_{S}\right)\left(-\kappa_{\gamma}+\Omega\right),
$$


(4) motion by anisotropic surface diffusion,

$$
v=\nabla_{S} \cdot D \nabla_{S} \kappa_{\gamma},
$$

where $\nabla_{S}$ denotes both the gradient and the divergence restricted to the surface $S$.

Observe that since $\kappa_{\gamma}$ is the $L^{2}$ gradient of surface free energy, motion law (3), when $\Omega=0$, is gradient flow in the $L^{2}$ inner product, weighted by $\frac{1}{M}$. Motion law (4) arises as follows: the weighted mean curvature acts as a potential, the flux of atoms is down the gradient of the potential with diffusion coefficient $D$, and the divergence of the flux gives the rate of accumulation of material, which is the normal growth rate. Since

$$
\delta_{E} S(\mathbf{g})=\int_{S} \kappa_{\gamma} \mathbf{n} \cdot \mathbf{g} d \mathcal{H}^{2},
$$

if the integral of $\mathbf{g} \cdot \mathbf{n}_{S}$ is zero we can write

$$
\delta_{E} S(\mathbf{g})=\int \mathbf{g} \cdot \mathbf{n}_{S}\left(\Delta_{S}^{-1} \Delta_{S} \kappa_{\gamma}\right) d \mathcal{H}^{2},
$$

where $\Delta_{S}$ is the standard surface Laplacian and $\Delta_{S}^{-1} f$ is the unique function satisfying $\Delta_{S}\left(\Delta_{S}^{-1} f\right)=f$. Since the $H^{-1}$ inner product of $f$ and $g$ (where $f$ and $g$ are each assumed to have integral zero) is $-\int_{S} g \Delta_{S}^{-1} f$, surface diffusion is in fact gradient flow in the $H^{-1}$ inner product on $S$, weighted by $\frac{1}{D}$. Like motion by weighted mean curvature, it decreases $E$ fastest, but with a different notion of distance. There are also motion laws combining attachment-detachment and surface-diffusion kinetics; they turn out to be gradient flow with respect to the sum of the inner products above on the space of normal vector fields with integral zero. See TC1] for a discussion of this and the next topic.

These "sharp interface" models have "diffuse interface" analogs. In diffuse interface models at each time $t$ there is an order-parameter or phase-field $\phi(t,):. R^{3} \rightarrow R$ which is supposed to take value 1 inside the crystal and -1 outside it; the interface is the region of transition between these values (which might for the sake of specificity be taken to be where $\phi$ is between .7 and -.7 ). There are several ways to deal with polycrystals, involving either an additional orientation field or order parameters that take values in a higher dimensional space. The energy of a given function $\phi$ can be modeled as

$$
E(\phi)=\int_{R^{3}}\left[\frac{\epsilon}{2}(\Gamma(\nabla \phi))^{2}+\frac{1}{\epsilon} F(\phi)\right] d \mathcal{L}^{d},
$$

where $\Gamma$ is a continuous, positive (except at 0 ) and positively homogeneous function on $R^{3}$, and $F$ is a double-well potential energy function such as $F(\phi)=\left(1-\phi^{2}\right)^{2}$ or $(1-u) \log (1-u)+(1+u) \log (1+u)$. The isotropic case is just $\Gamma(\nabla \phi)=|\nabla \phi|$. The energy $E$ can in this case be recognized as the scalar version of the relaxed energy used to study generalized minimizers of the Dirichlet integral $\int|\nabla \phi|^{2}$, when $\phi$ takes values in the unit $n$-sphere. If $\phi$ varies only in the direction of a given vector $\mathbf{n}$, with $\lim _{x \cdot \mathbf{n} \rightarrow-\infty} \phi(x)=-1$ and $\lim _{x \cdot \mathbf{n} \rightarrow \infty} \phi(x)=1$, then the width of the interfacial region for an equilibrium solution is proportional to $\epsilon$ and has energy per unit area along the interface of $\gamma(\mathbf{n})=c_{F} \Gamma(\mathbf{n})$, where $c_{F}=\int_{-1}^{1} \sqrt{2 F(\phi)} d \phi$. In case $F(\phi)=\left(1-\phi^{2}\right)^{2}$, any such equilibrium solution is a translation of $\phi(x)=$ $\tanh \left(\frac{x . \mathbf{n}}{\epsilon \Gamma(\mathbf{n})}\right)$. 
The Ginzberg-Landau equation is, in the anisotropic scalar- $\phi$ case, the anisotropic Allen-Cahn equation

$$
\phi_{t}=\mathcal{M}\left(-\frac{\delta E}{\delta \phi}\right)=\mathcal{M}\left(\epsilon \Delta_{\Gamma} \phi-\frac{1}{\epsilon} F^{\prime}(\phi)\right)
$$

where $\Delta_{\Gamma}$ is defined to be $\operatorname{div}_{x}\left(\left.\Gamma \nabla \Gamma\right|_{\mathbf{p}=\nabla \phi(x)}\right)$ and reduces to the usual Laplacian when $\Gamma(\mathbf{p})=|\nabla \mathbf{p}|$. As $\epsilon$ approaches zero, the solutions to this equation approach solutions to motion by weighted mean curvature, with $M=\mathcal{M} \epsilon \Gamma / c_{F}$. This has been shown by formal asymptotics in general (even in the case where $\Gamma$ is nondifferentiable in some directions), by gamma-convergence in the isotropic case, and in various intermediate degrees of rigor in intermediate cases. See, for example, GNS.

The isotropic Cahn-Hilliard equation is

$$
\phi_{t}=-\Delta\left(\epsilon \Delta \phi-\frac{1}{\epsilon} F^{\prime}(\phi)\right)
$$

and the anisotropic Cahn-Hilliard equation is

$$
\begin{gathered}
\phi_{t}=\nabla \cdot \mathcal{D} \nabla \frac{\delta E}{\delta \phi} \\
=\nabla \cdot \mathcal{D} \nabla\left(-\epsilon \Delta_{\Gamma} \phi+\frac{1}{\epsilon} F^{\prime}(\phi)\right) .
\end{gathered}
$$

This equation applies to phase separation, in the case where the phases just "fall apart" rather than nucleate and grow. Furthermore, if $\mathcal{D}$ is near zero when $\phi$ is not near zero, but is large for $\phi$ near zero, then solutions to the Cahn-Hilliard equation approximate surface diffusion, at least via formal asymptotics [GN], [NCE].

The objective is to prove theorems and devise computational methods which should be useful in applications, for these mathematical models (both sharp and diffuse) for the equilibrium and growth shapes of polycrystals. Significant issues are those of nondifferentiability of $\gamma$, singularities in $S$, and dealing with multijunctions like the triple junctions found in soap films. The mathematical questions include: What are the correct definitions? Do solutions exist? Are they unique? How regular are they, and what is the structure of any singularities? Computational questions include: appropriate parameterizations of surfaces, especially as shapes change; accuracy of numerical measurement of curvature and of second derivatives of curvature; and how both to detect the need to make and then to actually make topological changes (such as pinching off, or different pieces of surface colliding, or different triple junctions or point junctions colliding).

To illustrate the importance of interfaces to materials science, Table 1 presents the program of the Solid States Studies in Ceramics Gordon Research Conference which took place the same week as the Mathematical Challenges meeting, August 6$11,2000$.

2. Since the problem of proving that a given configuration of arbitrary polycrystals is minimizing can have many complications, we temporarily retreat to the simpler, isotropic case. (This challenge for soap bubble clusters is even more challenging for polycrystals with anisotropic surface energies.)

Challenge 2. Devise a means to prove that a given soap bubble cluster is areaminimizing under the constraint of separating regions of fixed given volumes from each other and from the outside. 
TABLE 1.

\section{Solid State Studies in Ceramics Gordon Research Conference http://mimp.mems.cmu.edu/ grc/ \\ Interfacial Networks in Polycrystals: Structure, Dynamics, and Control \\ August 6 to 11, 2000 - Kimball Union Academy - Meriden, NH \\ Chair: Gregory S. Rohrer • Vice-Chair: Stephen J. Bennison}

\begin{tabular}{|c|c|}
\hline $\begin{array}{l}\text { Sunday, PM } \\
\text { Turning Polycrystals to Single Crystals } \\
\text { Through Controlled Interface Motion } \\
\text { Discussion leader: Rajendra Bordia }\end{array}$ & $\begin{array}{l}\text { Martin Harmer, "Controlled Abnormal Grain Growth for } \\
\text { Solid State Crystal Conversion" } \\
\text { Amit Goyal, "Rolling Assisted Biaxially Textured } \\
\text { Substrates for the Growth of Single-Crystal-Like Ceramic } \\
\text { Superconducting Wires" }\end{array}$ \\
\hline $\begin{array}{l}\text { Monday, AM } \\
\text { Dynamic Properties of Interfaces } \\
\text { A.M. Cruickshank Award Lecture } \\
\text { Discussion leader: Sheldon Wiederhorn }\end{array}$ & $\begin{array}{l}\text { William W. Mullins, "Understanding and Controlling the } \\
\text { Motion of Neutral and Charged Grain Boundaries During } \\
\text { Normal and Abnormal Growth." } \\
\text { Janet Rankin, "Real-Time Observations of the Initial Stages } \\
\text { of Interface Evolution" } \\
\text { Doh-Yeon Kim, "Coarsening of Faceted Grains Dispersed in } \\
\text { a Liquid Matrix-Mechanism of Abnormal Grain }\end{array}$ \\
\hline $\begin{array}{l}\text { Monday PM } \\
\text { Electronic and Defect Properties of } \\
\text { Interfaces } \\
\text { Discussion leader: David Clarke }\end{array}$ & $\begin{array}{l}\text { Joachim Maier, MPI, " Size Effects on Point Defect } \\
\text { Distribution" } \\
\text { Xiaoqing Pan, University of Michigan, "Electronic } \\
\text { Properties of Grain Boundaries in } \mathrm{SnO}_{2} \text { Thin Films" }\end{array}$ \\
\hline $\begin{array}{l}\text { Tuesday, AM } \\
\text { Moving Boundaries } \\
\text { Discussion leader: Rowland M. Cannon }\end{array}$ & $\begin{array}{l}\text { John W. Cahn, NIST, "Testing the Fundamentals of Grain } \\
\text { Growth Theories with Simulations" } \\
\text { Lasar Shvindlerman, RWTH Aachen, "Grain Boundary } \\
\text { and triple Junction Motion: Current Results and Current } \\
\text { Problems" } \\
\text { Venkat Gopalan, Penn State, "Domain Wall Kinetics in } \\
\text { Ferroelectrics" }\end{array}$ \\
\hline $\begin{array}{l}\text { Tuesday PM } \\
\text { Grain boundary Sensitive Properties } \\
\text { Discussion leader: David Brandon }\end{array}$ & $\begin{array}{l}\text { Steve Bennison and Kurt Mikeska, "Property Control via } \\
\text { Grain Boundary Engineering in Alumina" } \\
\text { Preview of invited posters } \\
\text { Simone Peterson Hruda, Report from the Education Focus } \\
\text { Group (D. Birnie, G. Fischman, R. Riman, and R. Snyder) }\end{array}$ \\
\hline $\begin{array}{l}\text { Wednesday AM } \\
\text { Grain Boundary Properties } \\
\text { Discussion leader: Sunggi Baik }\end{array}$ & $\begin{array}{l}\text { Manfred Rühle, "Segregation, Pre-Wetting and Wetting at } \\
\text { Grain Boundaries in Metals and Ceramics" } \\
\text { Nigel Browning, "What Electronic Spectroscopy tells us } \\
\text { about Grain Boundary Structure Property Relationships" }\end{array}$ \\
\hline $\begin{array}{l}\text { Wednesday PM } \\
\text { Discussion Topic: The choice of length } \\
\text { scale in the study of grain boundary } \\
\text { networks: advantages and disadvantages of } \\
\text { the atomic and microscopic scales. }\end{array}$ & $\begin{array}{l}\text { Carol Handwerker, Discussion Leader } \\
\text { Viewpoints: Wayne King, C. Barry Carter, } \\
\text { Stephen Garofalini, Alain Karma }\end{array}$ \\
\hline $\begin{array}{l}\text { Thursday AM } \\
\text { Controlling Texture in Ceramics } \\
\text { Discussion Leader, Keith Bowman }\end{array}$ & $\begin{array}{l}\text { Gary Messing, "Texturing Ceramics via Templated Grain } \\
\text { Growth" } \\
\text { Desi Kovar, "Is there a pay-off for texturing ceramics?" } \\
\text { Arun Gokhale, "Effect of Gravity on Interfacial } \\
\text { Connectivity and 3D Coordination Number Distribution in } \\
\text { Liquid Phase Sintered Microstructures" }\end{array}$ \\
\hline $\begin{array}{l}\text { Thursday PM } \\
\text { Banquet Lecture }\end{array}$ & $\begin{array}{l}\text { Stephen Sass, "The Substance of Civilization: Materials and } \\
\text { Human History from the Stone Age to the Age of Silicon" }\end{array}$ \\
\hline
\end{tabular}

Reprinted by permission of Gregory S. Rohrer.

The study of soap films and soap bubbles has a glorious history. All of the following have worked on them, according to the article in the Encyclopedia Britannica by James Clerk Maxwell Max: Leonardo da Vinci, Isaac Newton, Leonhard Euler, J.L. Lagrange, Gaspard Monge, Pierre Laplace, Thomas Young, Carl Friedrich Gauss, Simeon-Denis Poisson, Joseph A.F. Plateau, William Thomson Lord Kelvin, Josiah W. Gibbs. Also, one of the first two Fields medals went to Jesse Douglas for his proof of the existence of a minimal surface with a prescribed boundary curve. Among those who have worked in this field since Douglas, special mention might be made of Richard Courant, for whom the Courant Institute at NYU is named, Herbert Federer, who wrote the major treatise on Geometric Measure Theory [F], and Fred Almgren, who developed Geometric Measure Theory to handle a large variety 
of variationally-formulated problems Alm99. Most of Almgren's graduate students have become leaders in the calculus of variations and/or the interdisciplinary work of mathematics and materials science: me, Jon Pitts, Harold Parks, Vladimir Scheffer, Ken Brakke, Frank Morgan, Robert Kohn, Brian White, Bruce Solomon, Dana Nance Mackenzie, Sheldon Chang, Kevin Perry, John Sullivan, John Steinke, Alice Underwood, David Caraballo, NungKwan (Aaron) Yip, and Kin Yan Chung.

The simplest case of the cluster question is: what is the shape of a single soap bubble? Proofs that it must be a round ball have been around for a century (e.g., via Steiner symmetrization). But only recently has it been proved that for the next simplest case of two regions of prescribed (not necessarily equal) volume, the minimizer is the expected standard double bubble, composed of two outer spherical caps and a third spherical cap separating them [HMRS], Mor00]. But for three or more regions of prescribed volume, where the expected answer constructed from portions of spheres is also well-known, there are no proofs at all. Among the things that are not known for multiple bubbles, even in the two-dimensional plane, are: Must each individual region within a cluster be connected? Must the "outside" region be connected, or could there be empty regions interior to the cluster? Must each region be simply connected?

What is known is the local structure of soap bubble clusters and films. Almgren defined an $(M, \epsilon, \delta)$-minimal set, which captures the idea of local force balance. He proved that configurations exist of regions with prescribed volumes and least area separating them from each other and from the outside, and that they are $(M, \epsilon, \delta)$-minimal sets. Finally, he proved that any $(M, \epsilon, \delta)$-minimal set is composed of smooth surfaces, each of constant mean curvature, except for a singular set of Hausdorff 2-dimensional measure zero [Alm76]. I simultaneously proved that for any $(M, \epsilon, \delta)$-minimal set, the singular set consists of smooth (Höldercontinuously differentiable) curves along which three surfaces meet at equal $\left(120^{\circ}\right)$ angles, together with isolated points at which four of those triple-junction curves come together as a smooth diffeomorphism of the cone over the edges of a regular tetrahedron T76.

A related problem is to find the minimum-area equal-volume partitioning of all of space. In $R^{2}$, it has long been thought to be the honeycomb, but only recently was it recognized as an open problem, and only still more recently was it solved. Specifically, Thomas Hales [Hal], [Mor00 proved: Let $C$ be a planar cluster of infinitely many regions of unit area. Then

$\lim \sup _{r \rightarrow \infty}($ Perimeter within $B(0, r)) /($ Area within $B(0, r)) \geq \rho_{0}$,

where $\rho_{0}$ is the limiting ratio for the unit regular hexagonal network.

Lord Kelvin found a partitioning of 3-space into equal volume regions by using identical 14-sided figures, the hexagonal faces of which are slightly curved in order to keep the $120^{\circ}$ angle criterion between adjacent surfaces. That partitioning remained the sole candidate for a minimizer for over 100 years, despite efforts to find a better partitioning. Finally, Weaire and Phelan used Ken Brakke's Surface Evolver program to compute a partitioning with two different equal-volume cells interlaced in what is known to physicists as a clathrate structure; they were able to show that it improved the area-to-volume ratio by about $1 \%$. Other structures have also been investigated, but none are better than this example. There is not yet any proof that this structure is optimal. Again, see [Mor00] for a discussion and references. 
The anisotropic problem equivalent to the single soap bubble problem is: given a surface free energy function $\gamma$, what is the solid of given volume having the smallest total surface energy $E(S)=\int_{x \in S} \gamma\left(\mathbf{n}_{S}(x)\right) d \mathcal{H}^{2} x$ ? The answer to that problem is $W_{\gamma}$, the result of the Wulff construction

$$
W_{\gamma}=\{x: x \cdot \mathbf{n} \leq \gamma(\mathbf{n}) \forall \mathbf{n}\} .
$$

My favorite proof of this is based on the divergence theorem (see [Mor00]). The shape of a crystal on a table is also known: use the Wulff construction, but in the direction $\mathbf{n}$ of the normal into the table, use the difference of the free energy per unit area of the crystal-table and the table-air in place of $\gamma(\mathbf{n})$. The following question is however completely open in $R^{3}$ : Is the shape of a crystal on a table with gravity convex? Results can be fairly easily obtained in $R^{2}$ for this and related problems (e.g., a centrally symmetric force rather than gravity acting on a crystal), but the 3 -dimensional problem has been a recognized open problem for at least 20 years. And no anisotropic versions of the double-bubble theorem in three dimensions have been proved.

3. Within the set of problems involving surface motion, I think an important problem is:

Challenge 3. Devise proofs and computations for motion by surface diffusion, including through topological changes and other singularities.

Many of the other presenters at the Mathematical Challenges meeting spoke about issues in partial differential equations, but only for second order equations. Motion by surface diffusion is a fourth order equation, whether in the diffuse interface approach where it is modeled by the Cahn-Hilliard equation (with a $\phi$ dependent diffusion coefficient), or the sharp interface approach where it is modeled by

$$
v=\nabla_{S} \cdot D \nabla_{S} \kappa_{\gamma}
$$

as described under Challenge 1.

In the sharp interface approach, methods such as viscosity solutions of HamiltonJacobi equations, which have been used to describe and calculate motion by mean curvature (see, for example, [OS] and [ES]), are not expected to work, because there is no maximum principle. Barrier arguments do not work.

Variational methods have been successful for proving all-time existence and properties of surface moving by weighted mean curvature, including ones with triple junctions and with an additional bulk driving force. In fact, there is a general variational approach to gradient flow of $E(S)$ with respect to an inner product $\langle$,$\rangle ,$ which uses the fact that the minimum over all $h$ of

$$
\langle\nabla E, h\rangle+\frac{1}{2 \Delta t}\langle h, h\rangle
$$

occurs at $h=-\nabla E$, together with

$$
\langle\nabla E, h\rangle \approx E\left(h_{\#} S\right)-E(S) .
$$

Therefore, setting $S(0)$ equal to the initial surface, for $i=0,1,2, \ldots$ one might set

$$
S((i+1) \Delta t)=h(i)_{\#}(S(i \Delta t)) \text {, }
$$

where $h(i)$ minimizes

$$
E\left(h_{\#} S(i \Delta t)\right)-E(S(i \Delta t))+\frac{1}{2 \Delta t}\langle h, h\rangle,
$$


and then try to obtain a limiting flow as $\Delta t$ approaches zero. I describe in some detail the application of this method to $L^{2}$ gradient flow for surface energy, and then the very minimal progress that has been made on $H^{-1}$ gradient flow.

In order to avoid having to assume normal vector fields exist (because of topological changes or other singularities or corners in $S$ ), it is better to replace the quantity being minimized by its approximate equal,

$$
E(\partial B)-E(\partial A)+\frac{1}{\Delta t} \int_{x \in B \sim A \cup A \sim B} \operatorname{dist}(x, \partial A) d \mathcal{L}^{3} x .
$$

Here $A$ and $B$ are regions, and $\partial A=S(i \Delta t)$. The boundary of $B$ is analogous to $h(i)_{\#} S(i \Delta t)$ but allows for more complicated geometries, including topological changes. Thus if $B$ is the minimizer, one sets $S((i+1) \Delta t)=\partial B$.

Solving these minimization problems clearly gives an approximation to motion by weighted mean curvature. The idea would be to apply it with $\Delta t=2^{-j}$, $j=1,2, \ldots$, and then show that there is a limit as $j$ goes to infinity.

One might try such a variational approach for a purely philosophical reason. Fred Almgren used to love to give the following quote by Euler: "For since the fabric of the universe is most perfect and the work of a most wise Creator, nothing at all takes place in the universe in which some rule of maximum or minimum does not appear." He would then follow it with a quote from Alfred North Whitehead: "It is a safe rule to apply that, when a mathematical or philosophical author writes with a misty profundity, he is talking nonsense." Unfortunately, I do not know the source of either quote. But a better reason for using this variational method is that it works:

Theorem ( $\mathrm{ATW}])$. Suppose the mobility $M$ is constantly 1, and that there are only two phases (or only two orientations in a polycrystal) and $\Omega=0$.

(1) Variationally-constructed flows by weighted mean curvature exist for all time and are a priori Hölder continuous in the mass topology (given by the metric which is the volume of the symmetric difference between regions).

(2) Shrinking Wulff shapes are barriers to motion by weighted mean curvature from both the outside and the inside.

(3) For smooth elliptic surface energies in any dimension, these variationallyconstructed flows by weighted mean curvature coincide with smooth flows (solutions to systems of partial differential equations) until the smooth flows develop singularities and cease to exist.

Theorem $([\mathrm{AT}])$. For crystalline surface energies in $R^{2}$, variationally constructed flows of curves by weighted mean curvature coincide with motion by crystalline curvature (solutions to coupled systems of ordinary differential equations, with prescribed means of passage through singularities as defined independently in [AG] and by me [T89], [T91, T93], with a computer generation of the motion illustrated in my paper and video in the compilation [COG]).

To include a constant mobility $M$ in this formulation, just divide the integrand by $M$. If $M$ is a function of the normal direction, then one should instead replace the integrand $\operatorname{dist}(x, \partial A)$ by $\inf _{y \in \partial A} M^{*}(x-y)$, where $M^{*}(\mathbf{p})=\sup _{\mathbf{q} \neq 0} \frac{\mathbf{p} \cdot \mathbf{q}}{M(\mathbf{q})}$. And to include a bulk driving force $\Omega$, include the extra terms $\frac{1}{\Delta t} \int_{x \in B \sim A} \Omega d \mathcal{L}^{3} x-$ $\frac{1}{\Delta t} \int_{x \in A \sim B} \Omega d \mathcal{L}^{3} x$. 

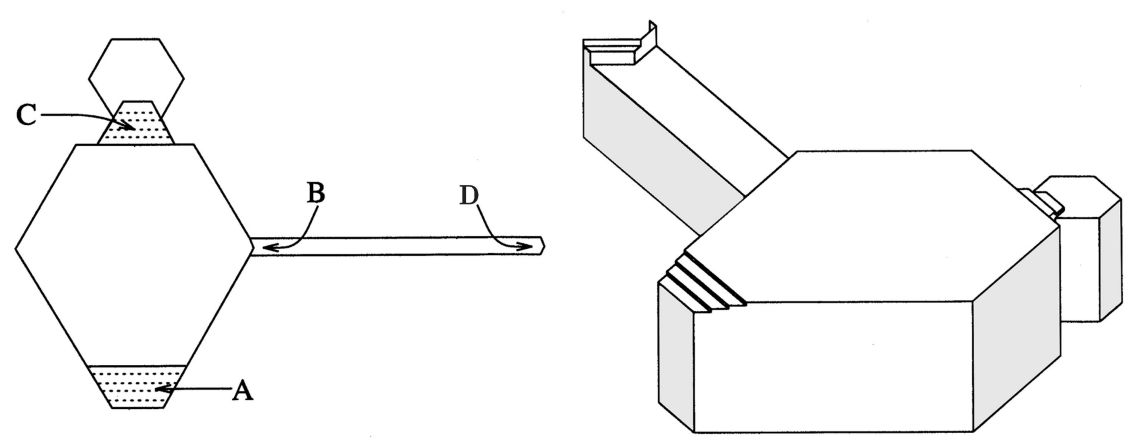

Figure 4. An illustration of the stepping of an initially flat facet due to motion by crystalline curvature. Steps are introduced at A, B, C and D. Courtesy of Jason Yunger Yun].

Theorem ([Yip]). The same theorems as in [ATW] hold with a normal-dependent mobility and a constant bulk driving force $\Omega$.

In order to prove a theorem analogous to that of $\left[\mathrm{AT}\right.$ for surfaces in $R^{3}$ moving by crystalline curvature, one needs an adequate definition of such motion. The key issue is that of "stepping" or creation of new facets, including continuous creation to give the infinitesimal corrugations known as varifolds [All. Several equivalent such definitions have only recently been found ([Yun, $[\mathrm{BNP}],[\mathrm{GG}]$ ). An algorithm for how to split arbitrary facets in motion of surfaces by crystalline weighted mean curvature has recently been derived [Yun] and is illustrated in Figure 4. Computations of surface motion by crystalline weighted mean curvature were published by me in 1992 [CCGW] on some surfaces that required stepping only along edges of facets.

The ATW formulation obviously applies only to single crystals (which however need not be connected or simply connected) and not to polycrystals, but there have been partial extensions to the polycrystalline case. Caraballo [Car produced the first partial extension, but his formulation cannot allow for a nonzero $\Omega$. On the other hand, [T99v extended the [ATW] formulation to planar polycrystals with crystalline surface free energies and/or extra constant $\Omega$; this formulation gives the previously derived crystalline results for triple junctions both when the surface free energy is nonzero [T93] and when $\gamma \equiv 0$ [T95], but has not yet been applied to prove general results such as ATW. (It should be noted that the results for zero $\gamma$ and for $\lim _{\epsilon \rightarrow 0} \epsilon \gamma$ are different, and may explain why the results of [RS] differ from those of [T95.)

Since surface diffusion is gradient flow with respect to the $H^{-1}$ inner product, there should be a variational formulation. An attempt to produce a volume-integral formulation similar to that of ATW, instead of the surface-integral formulation given above, was barely successful: existence of a limiting motion was proved, but without any proof that this motion is the same as surface diffusion when the surface is smooth [Chu. In fact, it took considerable effort to prove that any motion occurred at all! Because motion by surface diffusion is a fundamental law of surface motion, and because of this lack of any formulation which includes singularities, surface diffusion has been included as one of the major challenges. 
Crystalline surface diffusion of curves has been defined and programmed [CRCT, and some examples of computations are shown in Figure 5, along with examples of flows by $\kappa_{\gamma}$ and $\kappa_{\gamma}-\Omega$, where $\Omega$ is the average of $\kappa_{\gamma}$ over the curve. See my videos in [COG] and [CCGW] for animations.
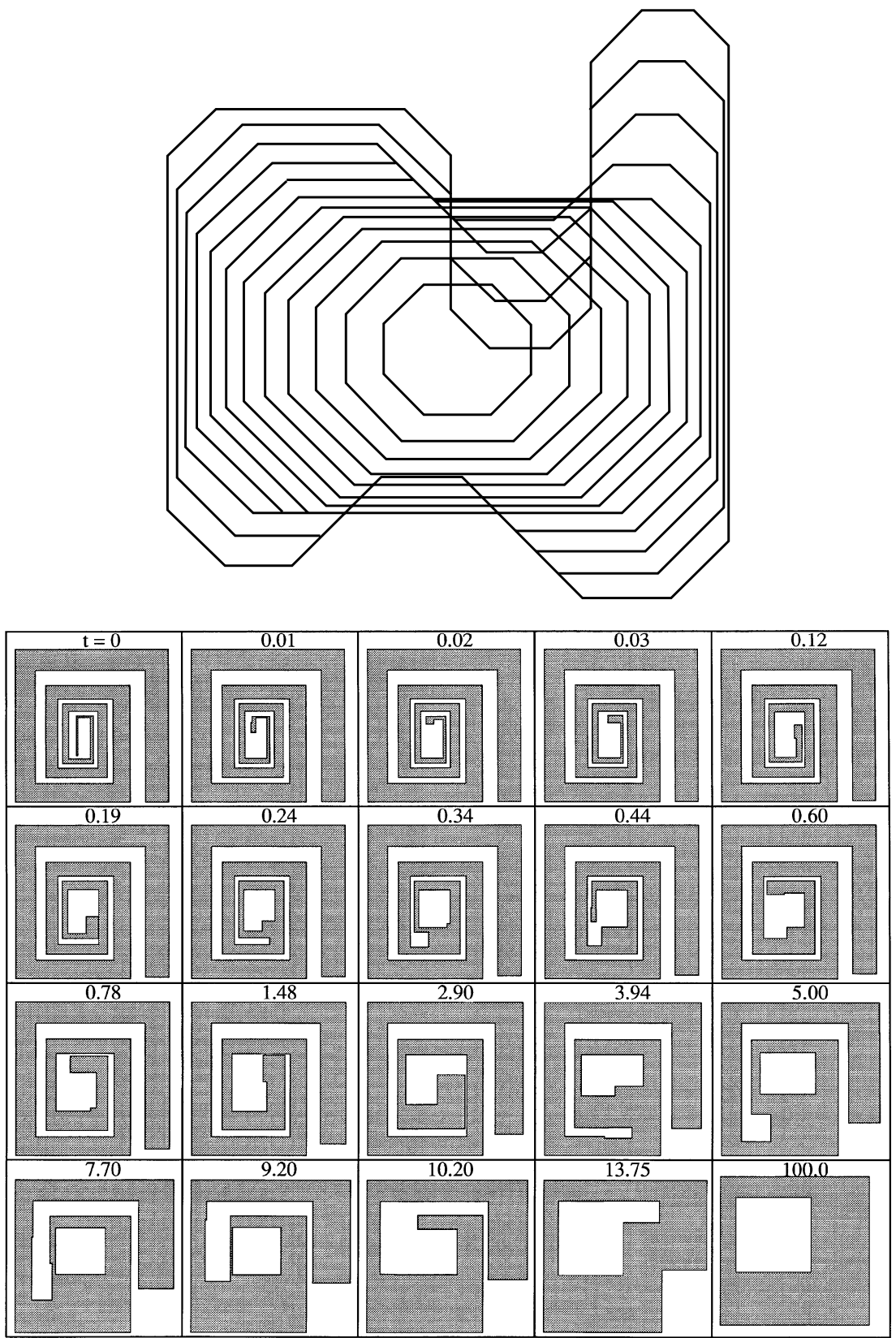

FiguRE 5a and b. Examples of motions of curves (a) by crystalline curvature, and (b) by crystalline surface diffusion. 


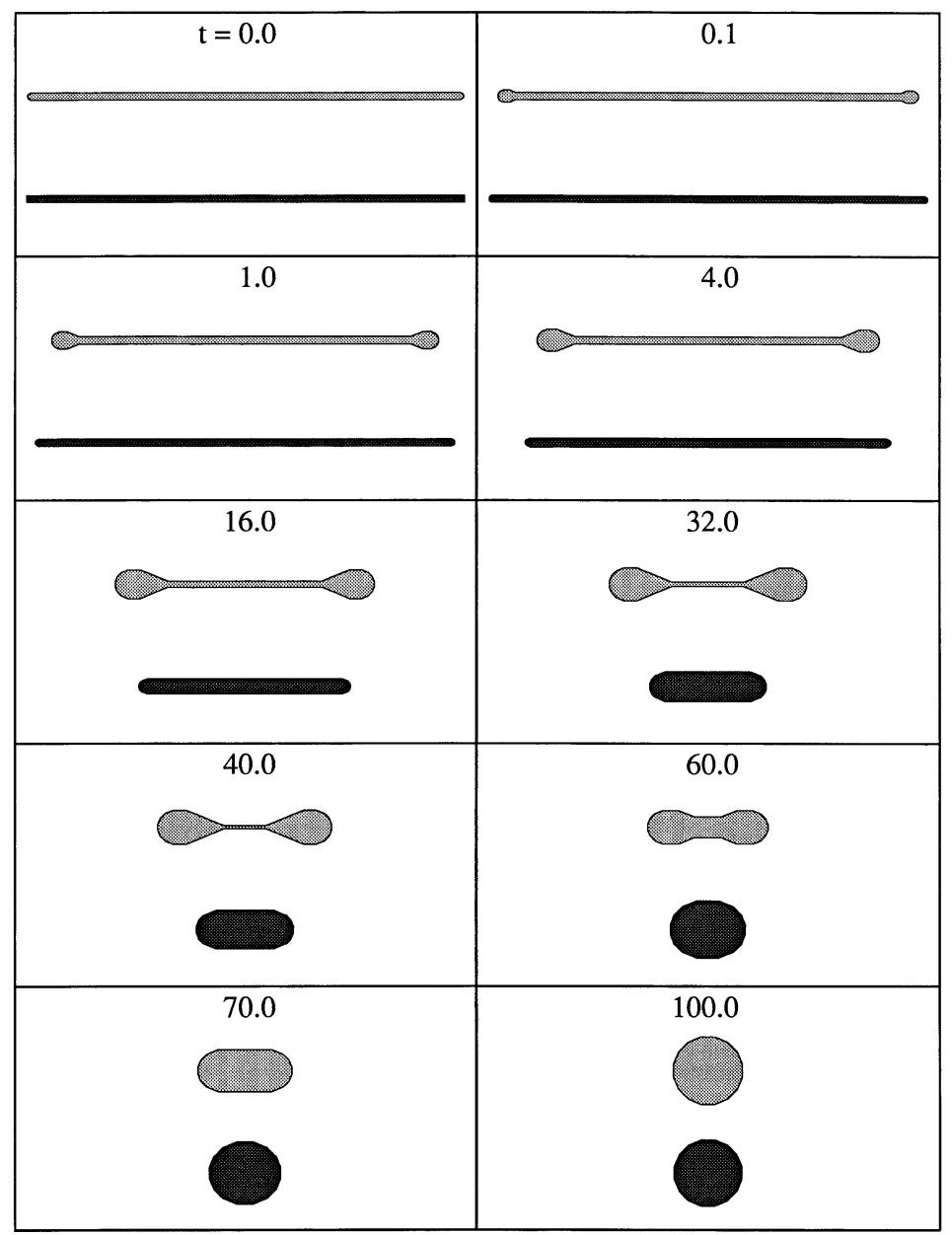

FiguRE 5c. Motion by surface diffusion is in grey and by curvature minus its average is in black.

4. The fourth major challenge I see is presented by the amazing development of computational power over the last few decades:

Challenge 4. Either find a way to extract meaningful models from the flood of simulations, or learn to use them in place of traditional models.

Often mathematicians think of models as follows: One starts with some data, devises a mathematical model to explain them, makes predictions from the models, checks the predictions against the previous data plus new data, refines the mathematical model, makes new predictions, etc. More and more often I am seeing people go directly from the data to the predictions without any intervening mathematical model.

For example, in an undergraduate text $\mathrm{Hab}$, a first order partial differential equation was derived for traffic flow, and as an application it was stated that traffic lights were installed at the Lincoln Tunnel to regulate the density and (according to the model) thereby increase the flow. So after a talk on June 5, 2000, at the 


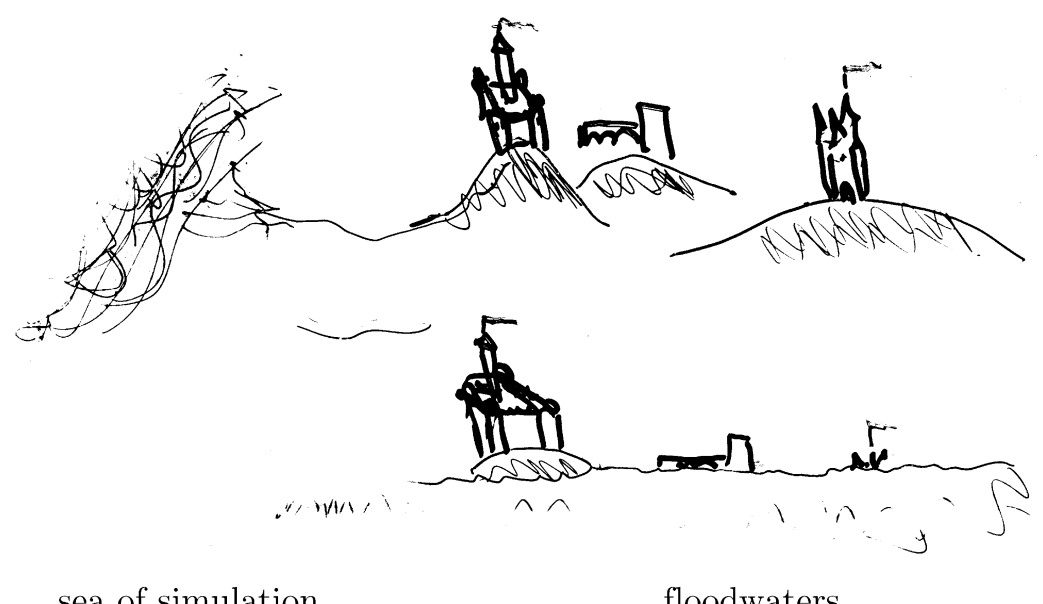

Figure 6. An illustration of the possible effect of the tidal wave of simulation on the castles of mathematical models.

New York Academy of Sciences, by Dr. Gregory Burnham, the Chief Information Officer of the New York-New Jersey Port Authority, I asked if this system was still in use, and in general whether any mathematical models such as PDE's were now used to manage traffic flow. His answer was no to both. The emphasis is on collection of traffic data (e.g., via E-Z Pass) and its processing to help manage traffic. In other words, it seems as if the step of mathematical modeling has been skipped; the connection from data to predictions and back is direct. And Dr. Burnham received his doctorate in mathematics from Northwestern University!

Perhaps mathematical modelling will also be skipped in the use of data from simulations to control manufacturing processes. Rather than achieving understanding based on theories such as I've discussed, there may be direct contact between the simulations and the processing. This is not really new; there has always been an art to doing metallurgy and especially ceramics. It is true that the space of possible manufacturing processes is very high-dimensional; but as David Donoho discussed in his delightful lecture at the AMS Math Challenges meeting at UCLA in August 2000 , there can be ways to efficiently extract patterns from high dimensional data sets.

I see the scene somewhat as in Figure 6. Currently there are castles of mathematical modeling on various hills, with well-controlled streams of computations emerging from them. On the horizon is a tidal wave, which threatens to inundate and submerge the castles in a sea of simulation. And I see four possible ways to cope with the flood.

First, one could seek high ground, above the flood. Here, one could continue to construct mathematical models in the traditional way; clearly, it is important to choose problems that will not be swamped by the simulation wave.

Secondly, one could dive in and swim: that is, one could try to devise simulations that are faster and more accurate and which give more information.

Thirdly, one could wait for the rich loam that is usually deposited by floods. An example of this is the computational method devised by Art Voter V]. Here, the 
desire is to compute global minima without getting stuck in local minima of an energy profile. The new idea is to "raise all the valleys" of the energy landscape, skate rapidly across any resulting flat areas, then lower back to the original landscape. Little mathematical analysis of this technique has been done.

Fourthly, one could learn to fish - to extract protein from the sea. Perhaps applying developing techniques such as those given by Donoho is the most effective way to make progress.

An interesting example of the impact of computations is the atomic-level simulation of evolving cylindrical grains inside other crystals, in a three-dimensional box with periodic boundary conditions. Grains are observed not just to shrink but also, if the misorientation is initially small, to rotate to a higher misorientation (with a higher surface energy per unit area, but a lower total surface energy) [SC]. The rotation occurs because for small misorientations, the grain boundaries are in fact composed of dislocations, and these dislocations are conserved as the grain shrinks, leading to a higher density and hence a larger misorientation. On the other hand, for other misorientations, there is a superlattice common to both grains, and then sometimes the boundary can move by the coordinated motion of just a few atoms. In this case there appears to be no grain rotation.

What does a mathematician do with such a simulation? Continue to study motion by weighted mean curvature for its own intrinsic beauty (and hope there are other situations where it is a more adequate model)? Decide that only atomiclevel simulations can predict how materials will behave, and investigate the behavior by doing more simulations? Try to construct a new continuum model which will incorporate this behavior? Search over all such simulations for other effects? My own inclination has been to try to construct new continuum models [TC2]. But having said this, I would like to point out some computational schemes that are still under development but already proving to be very useful.

The first is Ken Brakke's Surface Evolver program [B]. Developed as a project of The Geometry Center, it was used to compute the partitioning of space mentioned above that beat Lord Kelvin's example, and it has been used to compute shapes of soap films and bubble-film combinations on wire frames (see the minimal surface teams' video as Almgren's contribution to the COG compilation). It is also being used to compute the shapes of liquid solder in constructing electronic circuit boards, as part of the solder interconnect project of the Center for Theoretical and Computational Materials Science (CTCMS) at NIST; see the website www.ctcms.nist.gov. It has been used to compute 2-dimensional grain growth in the plane (e.g., Brakke's video in [CCGW]) and to compute where the fuel goes in fuel tanks in the Space Shuttle Teg]. It has also been used in basic mathematics to compute minimal surfaces with given symmetries $[\mathrm{B}$, to work on the Willmore Conjecture about surfaces minimizing the integral of the square of the mean curvature [HKS, and to compute minimal surfaces of higher dimension and codimension [B]. Furthermore, Alice Underwood proved in her Princeton University Ph.D. thesis (1993) that one can provide an accuracy certificate for some kinds of computations. (The conditions derived in that proof seem too stringent to be very useful.)

Another CTCMS project is called OOF, for object-oriented finite element analysis of real material microstructures. Here one starts with a micrograph, selects features in the image and assigns material properties to them, performs a virtual experiment, and then measures and visualizes the internal stresses. It was named 
one of the top 25 Technologies of the Year by Industry Week magazine in December 1999. Yet another CTCMS project uses phase field methods to compute compositional dendrites. Again, see www.ctcms.nist.gov.

5. Finally, since the subject of Geometric Measure Theory was created to define wider but still compact classes of objects to be regarded as "surfaces," and thus to enable one to use the direct method in the calculus of variations, I would like to close by mentioning some of its outstanding achievements and open problems, even though they may not be applicable to materials science.

The first achievement is the definition of the spaces $\mathbf{I}_{m}$ of integral currents in $R^{n}$, by Federer and Fleming, and their use in homology and cohomology. The simplest way to define integral currents is as follows: Let $C_{m}$ be the set of bi-Lipschitz images of integral polyhedral $m$-chains in $R^{n}$, and for $T$ in $C_{m}$, let $\mathbf{N}(T)=\mathbf{M}(T)+\mathbf{M}(\partial T)$, where $\mathbf{M}(T)$ is the sum of $m$-dimensional areas of the images of the polyhedra times their multiplicities. Then $\mathbf{I}_{m}$ is the space-bounded $\mathbf{N}$-completion of $C_{m}$ in the space of currents, which are the dual space to the space of $C^{\infty}$ differential forms of compact support. (Federer uses a different definition but proves its equivalence via the Approximation Theorem [F, 4.2.20]). Similarly for integers $\nu \geq 2$, one can define $\mathbf{I}_{m}^{\nu}$, the space of integral flat chains modulo $\nu$, as the space-bounded $\mathbf{N}$ completion of $C_{m} / \nu C_{m}$, according to the mod $\nu$ Approximation Theorem (4.2.20) ${ }^{\nu}$ found in [F, 4.2.26].

Secondly, there are the various proofs that show that integral currents are the "right" way to consider minimal surfaces, rather than images of mappings of manifolds [W], Cha].

Thirdly, there is Almgren's massive paper which proves that any area-minimizing integral current of dimension $m$ in $R^{n}$ is regular except for a possible singular set of Hausdorff dimension no more than $m-2$. This last result circulated as a 1720 page preprint for many years, before being typed in $\mathrm{TeX}$ and put on the web by Vladimir Scheffer and recently published as one massive volume by World Scientific Press Alm00.

I believe the biggest open problem at the heart of Geometric Measure Theory is:

Challenge 5. Determine whether $\mathbf{I}_{m}^{\nu}$ is just $\mathbf{I}_{m} / \nu \mathbf{I}_{m}$.

There is a counterexample to this conjecture in Federer's book, but it is wrong. Fred Almgren had a hand-written proof outline that the conjecture was correct when he died, but there is in it an assertion whose proof is labeled "missing" and for which I have found a counterexample (verified by Enrico Bombieri). Therefore the conjecture has to be considered open once again.

\section{REFERENCES}

[All] W. K. Allard, On the first variation of a varifold, Annals of Math. 95 (1972), 417-491. MR 46:6136

[Alm76] Frederick J. Almgren, Jr, Existence and regularity almost everywhere of solutions to elliptic variational problems with constraints, Memoirs Amer. Math. Soc. 4165 (1976) viii + 199 pages. MR 54:8420

[Alm99] Selected Works of Frederick J. Almgren, Jr., American Mathematical Society, 1999. MR 2001f:01053

[Alm00] Frederick J. Almgren, Jr., Almgren's Big Regularity Paper: Q-valued functions minimizing Dirichlet's integral and the regularity of area minimizing rectifiable currents up to codimension two, Vladimir Scheffer and Jean E. Taylor, editors, World Scientific Press, 2000 
[AT] Fred Almgren and Jean E. Taylor, Flat flow is motion by crystalline curvature for curves with crystalline energies, J. Differential Geometry 42 (1995), 1-22. MR 96h:58034

[ATW] Fred Almgren, Jean Taylor and Lihe Wang, Curvature Driven Flows: A Variational Approach, SIAM Journal of Control and Optimization 31 (1993), 387-438. MR 94h:58067

[AG] S. Angenent and M. Gurtin, Multiphase thermomechanics with interfacial structure. 2. Evolution of an isothermal interface. Arch Rat. Mech. Anal 108 (1989) 323-391. MR 91d:73004

[BNP] G. Bellettini, M. Novaga, and M. Paolini, Facet-breaking for three dimensional crystals evolving by mean curvature, Interfaces and Free Boundaries, 1 (1999), 39-55.

[B] Kenneth A. Brakke, The surface evolver, Experiment. Math. 1 (1992), no. 2, 141-165. MR 93k:53006

[CH] J. W. Cahn and D. W. Hoffman, A vector thermodynamics for anisotropic surfaces II. Curved and faceted surfaces, Acta Met. 22 (1974) 1205-1214.

[Car] David Caraballo, thesis, Princeton University, 1996.

[CRCT] Craig Carter, Andrew Roosen, John Cahn, and Jean E. Taylor, Shape evolution by surface diffusion and surface attachment limited kinetics on completely faceted surfaces, Acta Metal. Mater. 43 (1995),4309-4323.

[Cha] Sheldon Chang, Two dimensional area minimizing integral currents are classical minimal surfaces, J. Amer. Math. Soc. 1 (1988), 699-778. MR 89i:49028

[Chu] Kin Yan Chung, Ph.D. thesis, Princeton University, 1997.

[CCGW] Computational Crystal Growers Workshop (Jean E. Taylor, ed.), Selected Lectures in Mathematics, American Mathematical Society (1992). MR 94f:58007

[COG] Computing Optimal Geometries, J. E. Taylor, ed., Selected Lectures in Mathematics, Amer. Math. Soc. 1991. MR 93a:65021

[ES] Lawrence C. Evans and Joel Spruck, Motion of Level Sets by Mean Curvature I, II, III, and IV, in (respectively) J. Differential Geometry 33(1991), 635-681; Trans. Amer. Math. Soc. 330 (1992), no. 1, 321-332 J. Geom. Anal. 2 (1992), no. 2, 121-150; J. Geom. Anal. 5 (1995), no. 1, 77-114. MR 92h:35097 MR 92f:58050 MR 93d:58044, MR 96a:35077

[F] H. Federer, Geometric Measure Theory, Springer-Verlag, New York, 1969. MR 41:1976

[GG] M.-H. Giga and Y. Giga, Evolving graphs by singular weighted curvature, Arch. Rational Mech. Anal. 141 (1998), 117-198. (The paper by Yoshikazu Giga, Morton E. Gurtin, and José Matias, On the dynamics of crystalline motion, Japan Journal of Industrial and Applied Mathematics 15 (1998), 7-50, includes a comprehensive definition, but incorrectly "proves" that no stepping occurs.) MR 99j:35118 MR 99h:73008

[GN] Harald Garcke and Amy Novick-Cohen, A singular limit for a system of degenerate Cahn-Hilliard equations, Adv. Differential Equations 5 (2000), no. 4-6, 401-434. MR 2001e:35097

[GNS] Harald Garcke, Britta Nestler, and Barbara Stoth, On anisotropic order parameter models for multi-phase systems and their sharp interface limits. Phys. D 115 (1998), no. 1-2, 87-108. MR 99g:82023

[Hab] R. Haberman, Mathematical Models, Prentice-Hall, Inc., Engelwood Cliffs, NJ, 1977. Pages 257-394 deal with traffic flow, through the use of characteristics with shocks and fans. MR 55:14066

[Hal] Thomas C. Hales, The honeycomb conjecture. Discrete Comput. Geom. 25 (2001), no. 1, 1-22. MR 2002a:52020

[HC] D. W. Hoffman and J. W. Cahn, A vector thermodynamics for anisotropic surfaces I. Fundamentals and application to plane surface junctions, Surf. Sci. 31 (1972) 368-388.

[HKS] Lucas Hsu, Rob Kusner, and John Sullivan, Minimizing the squared mean curvature integral for surfaces in space forms, Experiment. Math. 1 (1992), no. 3, 191-207. MR 94a:53015

[HMRS] Michael Hutchings, Frank Morgan, Manuel Ritoré, and Antonio Ros, Proof of the double bubble conjecture, Annals of Math. 155 (2002), 459-489.

[Max] James Clerk Maxwell, Capillary Action, in Encyclopaedia Britannica, 11th Edition, 5, 256-275. 
[Mor00] Frank Morgan, Geometric Measure Theory: A Beginner's Guide, Third Edition, 2000. Academic Press, Inc., San Diego, CA, 2000. MR 2001j:49001

[Mor94] Frank Morgan, Clusters minimizing area plus length of singular curves. Math. Ann. 299 (1994), no. 4, 697-71. MR 95g:49083

[NCE] Amy Novick-Cohen, J. W. Cahn, and C. M. Elliott, "The Cahn-Hilliard equation: motion by the Laplacian of the mean curvature," Euro. J. Appl. Math., 7, 287-301 (1996). MR 97g:80010

[OS] Stan Osher and James A. Sethian, Fronts propagating with curvature-dependent speed: algorithms based on Hamilton- Jacobi formulations. J.Comput.Phys. 79 (1988), no. 1, 12-49. MR 89h:80012

[RS] Fernando Reitich and H. Mete Soner, Three-phase boundary motions under constant velocities. I. The vanishing surface tension limit, Proc. Roy. Soc. Edinburgh Sect. A 126 (1996), no. 4, 837-865. MR 97h:73012

[SC] S.G. Srinivasan and J.W. Cahn, Challenging some free-energy reduction criteria for grain growth, preprint.

[T76] Jean E. Taylor, The structure of singularities in soap-bubble-like and soap-film-like minimal surfaces, Annals of Math. 103 (1976), 489-539. MR 55:1208a

[T78] Jean E. Taylor, Crystalline variational problems, Bull. AMS 84 (1978), 568-588; Mean curvature and weighted mean curvature, Acta Metall. Mater. 40 (1992), 1475-1485. MR 58:12649

[T89] Jean E. Taylor, Crystals, in equilibrium and otherwise, video tape of AMS-MAA joint lecture, Boulder, CO 1989, American Mathematical Society, Providence, RI. MR 92c:58019

[T91] Jean E. Taylor, Constructions and Conjectures in Crystalline Nondifferential Geometry, in Differential Geometry, B. Lawson and K. Tenenblat, eds, Pitman Monographs and Surveys in Pure and Applied Math 52 (1991), 321-336. MR 93e:49004

[T93] Jean E. Taylor, Motion of curves by crystalline curvature, including triple junctions and boundary points, Differential Geometry, Proceedings of Symposia in Pure Math. 51 (part 1) (1993), 417-438. MR 94c:53012

[T95] Jean E. Taylor, The motion of multiple-phase junctions under prescribed phaseboundary velocities, J. Diff. Eq. 119 (1995), 109-136. MR 96e:73013

[T99m] Jean E. Taylor, Mathematical Models of Triple Junctions, Interface Science 7 (1999), 243-250.

[T99v] Jean E. Taylor, A Variational Approach to Crystalline Triple Junction Motion, J. Stat. Phys. 95 (1999), 1221-1244. MR 2000i:74073

[TC1] Jean E. Taylor and J. W. Cahn, Linking Anisotropic Sharp and Diffuse Surface Motion Laws via Gradient Flows, J. Stat. Phys. 77 (1994), 183-197. MR 95j:58029

[TC2] Jean E. Taylor and John W. Cahn, On motion-induced rotation of embedded crystals, preprint.

[Teg] James Tegart, Three-Dimensional Fluid Interfaces in a Cylindrical Container, in Computing Optimal Geometries, J. E. Taylor, ed., Selected Lectures in Mathematics, Amer. Math. Soc. 1991. MR 93a:65021

[V] Arthur F. Voter, Hyperdynamics: Accelerated molecular dynamics of infrequent events, Phys. Rev. Lett. 78 (1997), 3908-3911.

[W] Brian White, Existence of least-area mappings of $N$-dimensional domains. Ann. of Math. (2) 118 (1983), no. 1, 179-185; Mappings that minimize area in their homotopy classes. J. Differential Geom. 20 (1984), no. 2, 433-446. MR 85e:49063 MR 86f:49107

[Yip] NungKwan Yip, Ph.D. thesis, Princeton University, 1996. Also see: Stochastic motion by mean curvature. Arch. Rational Mech. Anal. 144 (1998), no. 4, 313-355. MR 99m:60100

[Yun] Jason Yunger, Ph.D. thesis, Rutgers University, 1998.

Department of Mathematics, Rutgers University, Piscataway, New Jersey 08855

E-mail address: taylor@math.rutgers.edu 